\title{
Center of Mass Estimation for a Spinning Spacecraft Using Doppler Shift of the GPS Carrier Frequency
}

\author{
Joseph E. Sedlak ${ }^{1}$ \\ a. i. solutions, Inc., 4500 Forbes Blvd., Suite 300, Lanham, MD, 20706, USA
}

\begin{abstract}
A sequential filter is presented for estimating the center of mass $(\mathrm{CM})$ of a spinning spacecraft using Doppler shift data from a set of onboard Global Positioning System (GPS) receivers. The advantage of the proposed method is that it is passive and can be run continuously in the background without using commanded thruster firings to excite spacecraft dynamical motion for observability.

The NASA Magnetospheric Multiscale (MMS) mission is used as a test case for the CM estimator. The four MMS spacecraft carry star cameras for accurate attitude and spin rate estimation. The angle between the spacecraft nominal spin axis (for MMS this is the geometric body $Z$-axis) and the major principal axis of inertia is called the coning angle. The transverse components of the estimated rate provide a direct measure of the coning angle. The coning angle has been seen to shift slightly after every orbit and attitude maneuver. This change is attributed to a small asymmetry in the fuel distribution that changes with each burn. This paper shows a correlation between the apparent mass asymmetry deduced from the variations in the coning angle and the CM estimates made using the GPS Doppler data. The consistency between the changes in the coning angle and the CM provides validation of the proposed GPS Doppler method for estimation of the CM on spinning spacecraft.
\end{abstract}

\section{Introduction}

The NASA Magnetospheric Multiscale (MMS) mission consists of four nearly identical spinning spacecraft in highly elliptical orbits flying in a tight formation. The orbital period during the first science phase of the mission is approximately 24 hours and the spin rate is 3.1 revolutions per minute (rpm). The mission is designed to study magnetic reconnection and related phenomena in the plasma of the Earth's magnetosphere. Maintenance of the formation requires very accurate knowledge of the four orbits. For this reason each of the MMS spacecraft carries four primary Global Positioning System (GPS) Navigator receivers, plus four backup receivers. MMS uses these GPS receivers and an Ultra-Stable Oscillator in conjunction with the Goddard Enhanced Onboard Navigation System (GEONS) software to determine the spacecraft orbits and to satisfy the mission time determination requirements, as described in Ref. 1.

Reference 2 presents the design for the MMS Attitude Ground System (AGS). The AGS exists as one component of the Flight Dynamics Operations Area of the MMS Mission Operations Center. It has responsibility for the following functions:

- Validating the onboard estimates of the attitude and spin vector,

- Calibrating the attitude sensors, accelerometer bias, center of mass, and spacecraft inertia tensor,

- Planning spin axis targets and predicting the spin precession from environmental torques as part of the overall maneuver planning effort, and

- Generating a definitive attitude and spin rate history for use by the science teams.

The spacecraft center of mass $(\mathrm{CM})$ is one of the many input parameters needed for precision navigation. Knowledge of the CM is necessary for determining the moment arm and resultant torques when thrusting. Error in CM knowledge leads to unintended torques and a build-up of angular momentum that subsequently must be unloaded with additional burns. Prelaunch inertia measurements provided by the NASA Goddard Space Flight Center, plus the geometry of the deployable booms have been used to model the CM and inertia tensor as a function of boom

${ }^{1}$ Email: joseph.sedlak@ai-solutions.com

SPACE 2016, AIAA/AAS Astrodynamics Specialist Conference, Long Beach, California, September 2016. 
deployment and remaining fuel fraction. Using this information, the AGS team has defined an analytic model to provide the $\mathrm{CM}$ and inertia tensor for navigation applications for every maneuver. With this model as the starting point, improved on-orbit estimates of the inertial properties are expected to yield improved orbit maneuver performance and to reduce overall fuel usage.

It was realized during development of the MMS AGS that the Doppler shifts of the GPS carrier frequency observed in the four active GPS receivers could be used to estimate the spacecraft CM. The relative motion of each MMS and each GPS spacecraft contributes a large Doppler shift. This can be subtracted out since the orbital ephemerides are known. There will remain a small Doppler "ripple" due to the rotational motion of the MMS spacecraft carrying the individual GPS antennas in a circle. The corresponding instantaneous linear velocities of the antennas are a function of their vector distance from the MMS spacecraft CM. A sequential filter was devised to estimate the CM using this residual ripple of the Doppler shifts. ${ }^{2}$ Other methods for estimation of inertial properties require actively varying the spacecraft dynamics through thruster firings, and then combining thruster, accelerometer, and star camera data during and after these burns. The dynamic response of the spacecraft allows for observability of a larger state vector that includes thruster efficiencies as well as inertial properties. This "dynamic method" was applied with good success to MMS once since launch, prior to deployment of the four long, 60-meter wire booms. ${ }^{3}$ However, the advantage of the much simpler method proposed in this paper is that it is passive and can be run continuously in the background without commanded thruster firings.

This paper investigates the observed shift in the CM and changes in the inertia tensor using MMS flight data over a period of almost six months during which seven orbit and attitude maneuvers were performed. Section II presents the main details of the CM filter. Section III discusses how changes in the CM and inertia tensor could arise from an asymmetry in the fuel distribution. The presumed common cause for these changes implies a correlation that is investigated in Section IV, where the CM filter and inertia tensor results are presented and compared. Some brief conclusions are given in Section V.

\section{Center of Mass Filter}

The algorithm given in this section estimates the spacecraft CM using the Doppler shifts of the carrier frequencies from each of the GPS satellites being tracked by the onboard GPS system. This section reviews the basic algorithm and shows some early test results using simulated data. The flight data results are given in Section IV.

Each of the MMS spacecraft is equipped with four GPS receivers, plus four redundant backup receivers, which simultaneously track up to a total of 12 GPS spacecraft. These receivers provide data input to the GEONS software for onboard orbit determination. ${ }^{1}$ The AGS uses the Doppler shifts of the GPS carrier frequencies that are telemetered to the ground as a byproduct of the GEONS processing.

The GPS spacecraft are referred to as GPS space vehicles (SVs). For each SV, the relative motion between it and the MMS spacecraft causes a baseline mean Doppler shift. On top of this baseline there is a small ripple of the Doppler shift due to the MMS rotational motion that carries the GPS receiver towards and away from the SV. The amplitude of this sinusoidal variation of the Doppler shift is proportional to the projection of the rotational part of the antenna's velocity along the line towards the SV. Thus, fitting the Doppler signal gives us a measurement of that velocity component, which in turn is proportional to the spin rate times the vector from the true CM to the receiver. The difference between this measurement and the prediction based on the nominal CM is filtered to give the offset of the true CM from its nominal position. The attitude and all the orbital parameters are known, so the geometric parts of the measurement model are all fully determined except for the $\mathrm{CM}$ offset error.

The four GPS receivers are located approximately 1.6 meter from the spin axis and every 90 deg in azimuth about the MMS body. As MMS rotates about its Z-axis, the tracked SVs are handed off from one GPS antenna to the next. Thus, it is expected there will be good observability of the CM in the body $X-Y$-plane. However, there usually is no observability of the CM component along the spin axis. The exception is after attitude and orbit maneuvers when there is significant boom vibration and body nutation; this out-of-plane motion allows for some limited observability of the Z-component of the CM.

The MMS and GPS SV ephemerides provide the position and velocity vectors of the spacecraft CMs in the geocentric inertial (GCI) frame, $\vec{R}_{M M S}, \vec{V}_{M M S}, \vec{R}_{G P S}$, and $\vec{V}_{G P S}$. For the four GPS receivers on each MMS spacecraft, denote the $n^{\text {th }}$ GPS receiver location in the MMS body frame as $\vec{r}_{A n t, n}$. The nominal CM location in the MMS body 
frame is $\vec{r}_{C M 0}$, which is used as the a priori guess for the estimator. The true CM body frame position vector is denoted $\vec{r}_{C M}$ and its estimate is $\tilde{r}_{C M}$. The GCI position and velocity of the $n^{\text {th }}$ receiver are

$$
\vec{R}_{A n t, n}=\vec{R}_{M M S}+A^{T} \Delta \vec{r}
$$

and

$$
\vec{V}_{A n t, n}=\vec{V}_{M M S}+A^{T} \vec{\omega} \times \Delta \vec{r}
$$

where $\vec{\omega}$ is the rotation vector expressed in the body frame,

$$
\Delta \vec{r} \equiv \vec{r}_{A n t, n}-\vec{r}_{C M}
$$

and where the transpose of the attitude matrix, $A^{T}$, transforms vectors from the body frame to the GCI frame.

For convenience, define the following vector differences, where the attitude matrix transforms these from GCI to the body frame

and

$$
\Delta \vec{R} \equiv A\left(\vec{R}_{G P S}-\vec{R}_{M M S}\right)
$$

$$
\Delta \vec{V} \equiv A\left(\vec{V}_{G P S}-\vec{V}_{M M S}\right)
$$

The position and velocity of the GPS spacecraft relative to the $n^{\text {th }}$ receiver then can be expressed in the body frame as

and

$$
\vec{R} \equiv A\left(\vec{R}_{G P S}-\vec{R}_{A n t, n}\right)=\Delta \vec{R}-\Delta \vec{r},
$$

$$
\vec{V} \equiv A\left(\vec{V}_{G P S}-\vec{V}_{A n t, n}\right)=\Delta \vec{V}-\vec{\omega} \times \Delta \vec{r}
$$

With these definitions, the Doppler shift can be written as

$$
f^{\prime}=\gamma\left(1-\frac{\vec{V} \cdot \vec{R}}{c|\vec{R}|}\right) f
$$

where $\gamma=\left(1-V^{2} / c^{2}\right)^{-1 / 2}$. In the nonrelativistic limit, $\gamma$ is set to unity and the vector additions in Eqs. 6 and 7 do not make use of the Lorentz transformation or velocity addition formula. The fractional Doppler shift, $\mathrm{D}$, is defined to be

$$
D \equiv \frac{f^{\prime}-f}{f}=-\frac{\vec{V} \cdot \vec{R}}{c|\vec{R}|}
$$

The quantity $\mathrm{D}$ is the effective measurement for the CM estimator. The sensitivity matrix is derived from this quantity. The sensitivity matrix represents how the measurement varies with changes in the state vector, which in this case is the CM location in the body frame. Using the notation $[\vec{\omega} \times]$ for the cross-product operator,

$$
[\vec{\omega} \times] \equiv\left[\begin{array}{ccc}
0 & -\omega_{z} & \omega_{y} \\
\omega_{z} & 0 & -\omega_{x} \\
-\omega_{y} & \omega_{x} & 0
\end{array}\right],
$$

the inner product in the numerator of Eq. 9 is

$$
\begin{aligned}
\vec{V} \cdot \vec{R} & =(\Delta \vec{V}-[\vec{\omega} \times] \Delta \vec{r}) \cdot(\Delta \vec{R}-\Delta \vec{r}) \\
& =\Delta \vec{V} \cdot \Delta \vec{R}-([\vec{\omega} \times] \Delta \vec{r}) \cdot \Delta \vec{R}-\Delta \vec{V} \cdot \Delta \vec{r}+([\vec{\omega} \times] \Delta \vec{r}) \cdot \Delta \vec{r} .
\end{aligned}
$$


The denominator of Eq. 9 can be expanded to $1^{\text {st }}$-order in $\Delta \vec{r} /|\Delta \vec{R}|$ yielding

$$
\begin{aligned}
|\vec{R}|^{-1} & =[(\Delta \vec{R}-\Delta \vec{r}) \cdot(\Delta \vec{R}-\Delta \vec{r})]^{-1 / 2} \\
& \approx\left[\Delta R^{2}-2 \Delta \vec{R} \cdot \Delta \vec{r}\right]^{-1 / 2} \\
& \approx \Delta R^{-1}\left[1+\frac{\Delta \vec{R} \cdot \Delta \vec{r}}{\Delta R^{2}}\right]
\end{aligned}
$$

where $\Delta R$ is the magnitude of $\Delta \vec{R}$. The fractional Doppler shift becomes

$$
\begin{aligned}
D & \approx \frac{-1}{c \Delta R}[\Delta \vec{V} \cdot \Delta \vec{R}-([\vec{\omega} \times] \Delta \vec{r}) \cdot \Delta \vec{R}-\Delta \vec{V} \cdot \Delta \vec{r}]\left[1+\frac{\Delta \vec{R} \cdot \Delta \vec{r}}{\Delta R^{2}}\right] \\
& \approx \frac{-1}{c \Delta R}[\Delta \vec{V} \cdot \Delta \vec{R}-([\vec{\omega} \times] \Delta \vec{r}) \cdot \Delta \vec{R}-\Delta \vec{V} \cdot \Delta \vec{r}+\Delta \vec{V} \cdot \Delta \hat{R} \Delta \hat{R} \cdot \Delta \vec{r}],
\end{aligned}
$$

where $\Delta \hat{R}$ is the unit vector in the direction of $\Delta \vec{R}$.

The sensitivity matrix, $H$, is the partial derivative of the observation with respect to the state vector $\vec{r}_{C M}$. That is,

$$
H \equiv \frac{\partial D}{\partial \vec{r}_{C M}} \approx \frac{-1}{c \Delta R}\left[\Delta \vec{R}^{T}[\vec{\omega} \times]+\Delta \vec{V}^{T}-(\Delta \vec{V} \cdot \Delta \hat{R}) \Delta \hat{R}^{T}\right]
$$

Note that one minus sign comes from the partial derivative of Eq. 3, $\partial \Delta \vec{r} / \partial \vec{r}_{C M}=-I_{3}$. If one is solving for all three components of the CM position, then Eq. 14 gives the appropriate sensitivity matrix. However, the $Z$-component of the CM has very poor observability; it usually is preferred to solve only for the $X$-and $Y$-components. In this case, the sensitivity matrix is

$$
H_{x y} \equiv \frac{\partial D}{\partial \vec{r}_{C M}} \frac{\partial \vec{r}_{C M}}{\partial(x, y)}=\frac{\partial D}{\partial \vec{r}_{C M}}\left[\begin{array}{ll}
1 & 0 \\
0 & 1 \\
0 & 0
\end{array}\right] .
$$

This $H$ matrix is used in a sequential least-squares estimator to determine the value of $\vec{r}_{C M}$ from a set of measurements of the fractional Doppler shift, $D$. The CM remains constant between maneuvers, so no dynamic propagation or process noise is included as would be needed in a Kalman filter. In operations, this filter would not be used during maneuvers, but would be executed between maneuvers to determine any change in the CM.

For the initial preflight testing, the CM was given offset errors of $4 \mathrm{~cm}$ in the $X$ direction and $-4 \mathrm{~cm}$ in $Y$. The number of GPS SVs that are tracked by the MMS onboard navigation system varies with orbital position. This number peaks near perigee, and drops as MMS moves above the altitude of the GPS constellation. Based on preflight analysis, ${ }^{1}$ it was conservatively assumed for these tests that an average of seven SVs would be tracked for six hours centered on the MMS perigee and the data rate would be $1 \mathrm{~Hz}$. The simulated noise on the measured fractional Doppler shift was taken to be zero-mean, white, and Gaussian-distributed with a standard deviation of $10^{-9}$. (The actual flight data was much noisier and had a lower data rate, as is discussed in Sec. IV.) Figure 1 shows a typical result for the $X$-axis CM estimate under these test conditions (the $Y$-axis is similar). At the end of the run, the $X$-axis estimate is $3.51 \mathrm{~cm}$ and the $Y$-axis is $-4.10 \mathrm{~cm}$. The accuracies are consistent with the errors from the estimated covariance, shown as $1 \sigma$ error bounds in Fig. 1.

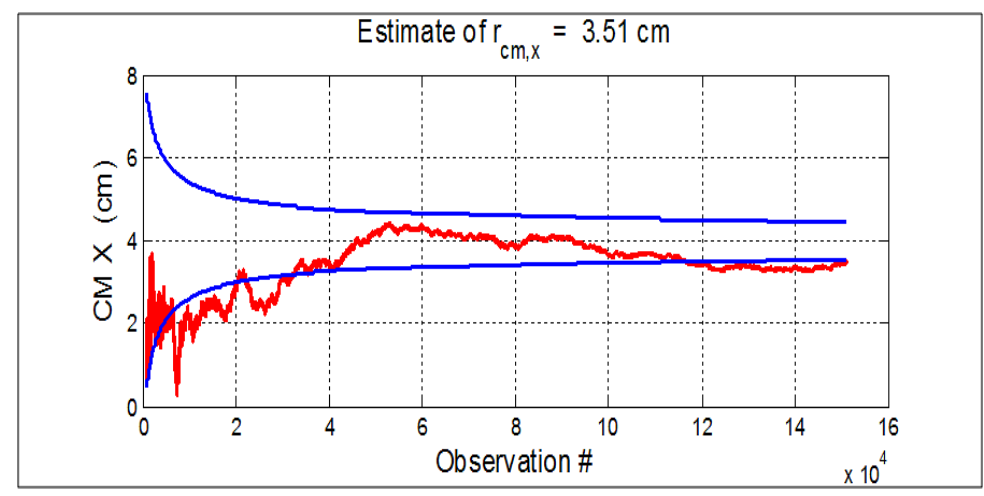

Figure 1. $X$-axis CM estimate (red) and $1 \sigma$ error bounds (blue) using MMS simulated data. The actual test value is $4 \mathrm{~cm}$. 


\section{Effect of Mass Shift on Center of Mass and Coning Angle}

This section gives a review of how the spacecraft $\mathrm{CM}$ and inertia tensor both change due to small mass asymmetries. The MMS propulsion system is designed to consume fuel uniformly from the four fuel tanks located symmetrically about the body spin axis, but some small error in this balance necessarily will accumulate with every maneuver. In general, mass asymmetry can arise from several sources, including non-uniform fuel usage, changes to the shapes of the diaphragms in the fuel tanks, or slightly uneven boom deployment lengths.

Changes to the mass distribution can change the off-diagonal elements of the spacecraft inertia tensor. This would lead to a change in the direction of the major principal axis (MPA) of inertia. ${ }^{4}$ If the changes in the CM and the MPA are both attributed to the same mass asymmetry, then they should be simply related through the expressions given below. (The MPA is the eigenvector of the inertia tensor having the largest eigenvalue. When all nutation and vibration have damped out, the spin axis will be aligned with the MPA. The angle between the nominal body Z-axis and the MPA is called the coning angle.)

The model used by the AGS for the spacecraft CM is based on prelaunch measurements with all booms stowed, an analytical model for deployed booms, and interpolation on the remaining mass of fuel in the tanks. At the time of this writing, the CM filter using GPS data described in the previous section is experimental and has not yet been used operationally for MMS support.

The model used for the spacecraft inertia tensor is also based on the prelaunch measurements, analytical model, and interpolation on the remaining fuel mass, but a coning angle correction is applied to this model after every maneuver. ${ }^{2}$ This coning calibration consists of an estimate of the true direction of the MPA in the body frame. The approach uses the high-accuracy star camera measurements to estimate the attitude and the direction of the angular momentum vector in GCI using an attitude and rate Kalman filter designed for spinning spacecraft. ${ }^{5}$ The MPA aligns, on average, with the angular momentum, which is constant in the inertial frame. Even when there are boom vibrations or nutation, the MPA rotates about the angular momentum vector, and the MPA can be estimated from the mean value of the angular momentum in the body frame as long as the averaging time span is sufficiently long (for MMS operations, four hours are allowed for these averages). The modeled inertia tensor then is rotated by a similarity transformation to agree with this in-flight estimate of the MPA, without making any other changes. This strategy has been shown to work well even shortly after maneuvers when the nutation and boom oscillations are large.

The main point being made here is that while the initial analytic models for the CM and inertia tensor do not account for mass asymmetry, the CM filter and the coning calibration are sensitive to the actual mass distribution. In particular, any deviation of the $\mathrm{CM}$ and coning angle from their analytic models should be attributable to the same mass asymmetry and will therefore be correlated.

The effects of coning are easily seen in the trending data collected daily for the MMS mission. Figure 2 shows the $X$ - and $Y$-axis components of the spin vector for MMS2. These are nonzero because the MPA is offset from the body $Z$-axis; the coning angle is roughly $0.13 \mathrm{deg}$. This causes the spin vector to project onto the body $X$ - and $Y$-axes. The changes seen in Fig. 2 occur at the times of maneuvers. Any asymmetry in the fuel usage from the four tanks, or changes in the shapes of the tank diaphragms, would cause inertia tensor changes resulting in changes like these seen in Fig. 2.

The rest of this section describes quantitatively how the $\mathrm{CM}$ and coning angle change as functions of the mass asymmetry.

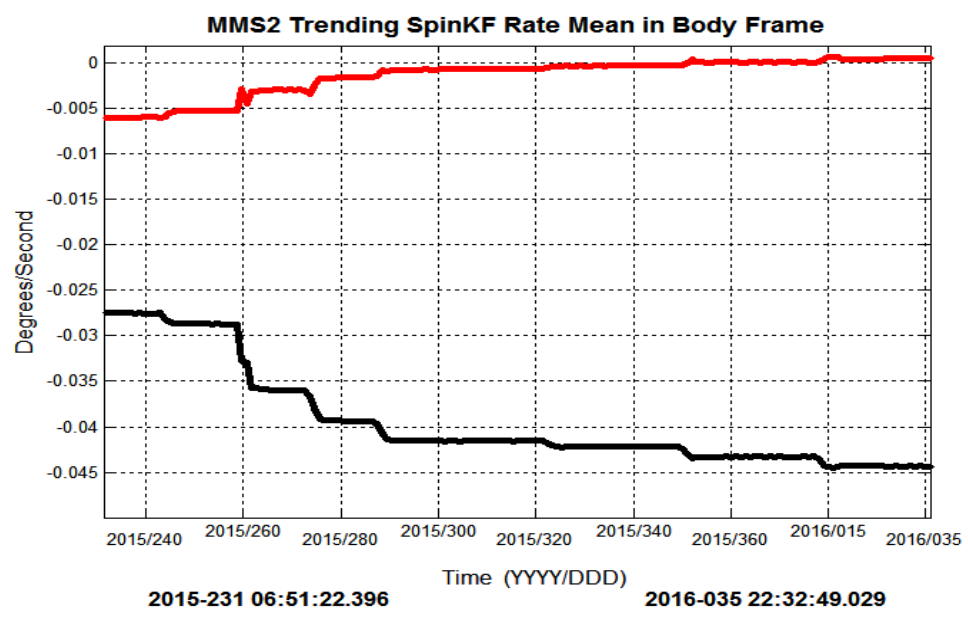

Figure 2. Body $X$ (red) and $Y$ (black) rotation rate components, plotted daily over five months. The spin rate is $18.6 \mathrm{deg} / \mathrm{sec}$.

The total fuel mass was $412 \mathrm{~kg}$ (103 kg per tank) and the spacecraft dry mass is $942 \mathrm{~kg}$, so the total initial spacecraft mass, $M_{\text {tot }}$, was $1354 \mathrm{~kg}$. The center of mass of each fuel tank is roughly $0.5 \mathrm{~m}$ from the spacecraft CM in the $X$ - $Y$-plane and near the spacecraft $\mathrm{CM}$ in the $Z$-direction. 
To begin to think about mass asymmetry, consider a quantity of fuel $\Delta m$ displaced from one tank to the tank on the opposite side along the body $Y$-axis. (The actual tank orientation does not matter since cylindrical symmetry will be assumed in the following for the unperturbed inertia.) The $Y$-axis distance of $\Delta m$ from the CM is $\Delta y \approx 0.5 \mathrm{~m}$. The spacecraft CM will shift by $2 \Delta y \Delta m / M_{\text {tot }}$, which is 0.074 centimeters for each kilogram shifted between tanks. (The factor of 2 appears because this imagines $\Delta m$ taken from one tank and that same $\Delta m$ added to the opposite tank.) More generally, one can show that shifting a mass $\Delta m$ by a vector $\Delta \vec{r}$ causes the CM to shift by $\left(\Delta m / M_{t o t}\right) \Delta \vec{r}$.

The corresponding change in the moment of inertia about the $Z$-axis for a shift of $\Delta m$ between fuel tanks is given by the parallel-axis theorem approximately as $M_{t o t}\left(2 \Delta y \Delta m / M_{t o t}\right)^{2}$, which is only $0.0007 \Delta m^{2} \mathrm{~kg}-\mathrm{m}^{2}$, with $\Delta m$ given in kilograms. The $Z$-axis moment of inertia is $5450 \mathrm{~kg}-\mathrm{m}^{2}$. This shows that the change in the moment of inertia about the MPA due to the change in the center of mass is negligible for this analysis.

With this same level of approximation, neither the $Z$-axis moment of inertia nor the transverse moment of inertia will change when the mass $\Delta m$ is shifted, because the same amount of mass still exists with the same moment arms (just shifted from one side to the other). However, the off-diagonal inertia elements will change in proportion to $\Delta m$. If the mass is shifted along the $Y$-axis of the body, then the $Y$-Z-component of the inertia tensor will change by $2 \Delta m$ times the offset of that mass in the $Y$ - and $Z$-directions from the center of mass. The $Y$-offset is taken to be the distance to the tank center, which is roughly $0.5 \mathrm{~m}$. For this analysis, assume that the mass $\Delta m$ is located near the center of one of the tank hemispherical caps. That would give $\Delta m$ an offset in the Z-direction of roughly $0.3 \mathrm{~m}$ from the spacecraft center of mass. Thus, the change in the off-diagonal component of the inertia tensor caused by the mass asymmetry $\Delta m$ is roughly

$$
I_{y z}=2 \Delta m \Delta y \Delta z \approx 0.3 \Delta m \mathrm{~kg}-\mathrm{m}^{2} \quad \text { (with } \Delta m \text { in } \mathrm{kg} \text { ). }
$$

This change in the off-diagonal element causes a change in the coning angle. This dependence is derived next under the assumption that that angle and $\Delta m$ are small (they will be found to be proportional). The coning angle can be determined by diagonalizing the inertia tensor. The rotation angle needed to do the diagonalization is the rotation from the old MPA to the new MPA. The old MPA is taken to be the body $Z$-axis. Thus, this rotation angle needed to perform the diagonalization is the coning angle.

Since only the effect of small changes in the mass are of interest here, the geometry is simplified by rounding the actual values, dropping the small initial off-diagonal terms, and averaging the transverse components. Then, the rigidbody inertia for the MMS spacecraft is approximately

$$
I_{\text {tot }}=\left[\begin{array}{ccc}
I_{t} & 0 & 0 \\
0 & I_{t} & 0 \\
0 & 0 & I_{z}
\end{array}\right]=\left[\begin{array}{ccc}
3240 & 0 & 0 \\
0 & 3240 & 0 \\
0 & 0 & 5450
\end{array}\right] \mathrm{kg}-\mathrm{m}^{2},
$$

With the contribution from Eq. 16, the perturbed inertia tensor is

$$
\tilde{I}=\left[\begin{array}{ccc}
I_{t} & 0 & 0 \\
0 & I_{t} & I_{y z} \\
0 & I_{y z} & I_{z}
\end{array}\right]
$$

This can be diagonalized by a rotation about the $X$-axis by some angle $\vartheta$, which is expressed using two rotation matrices since the inertia tensor is a 2 -index geometric object. That is,

$$
\left[\begin{array}{ccc}
I_{t} & 0 & 0 \\
0 & \tilde{I}_{t} & 0 \\
0 & 0 & \tilde{I}_{z}
\end{array}\right]=\left[\begin{array}{ccc}
1 & 0 & 0 \\
0 & c & s \\
0 & -s & c
\end{array}\right]^{T}\left[\begin{array}{ccc}
I_{t} & 0 & 0 \\
0 & I_{t} & I_{y z} \\
0 & I_{y z} & I_{z}
\end{array}\right]\left[\begin{array}{ccc}
1 & 0 & 0 \\
0 & c & s \\
0 & -s & c
\end{array}\right],
$$

where $s=\sin (\vartheta), c=\cos (\vartheta)$, and the tildes on the left-hand side of Eq. 19 indicate that the diagonal components may be affected. (They in fact change only in second-order, but that result is not needed for the current analysis.)

Considering just the (2,3)-element in Eq. 19, one finds

$$
0=\left(c^{2}-s^{2}\right) I_{y z}-s c\left(I_{z}-I_{t}\right) .
$$


This expression can be expanded into a quadratic equation in $s^{2}$ (since $c^{2}=1-s^{2}$ ). (Note that one solution of the quadratic is spurious since, for that case, the angle $\vartheta$ goes to 90 degrees when $I_{y z}$ goes to zero. The $Y$ - and $Z$-axes become reversed in that limit for that solution.) However, under the assumption that the angle $\vartheta$ is small, Eq. 20 can easily be solved in first-order to find

$$
\vartheta \approx s \approx \frac{I_{y z}}{I_{z}-I_{t}}
$$

Combining Eqs. 16, 17, and 21, one finds $\vartheta \approx 0.3 \Delta m / 2210$ (with $\Delta m$ in $\mathrm{kg}, \vartheta$ in radians). This yields a coning angle shift of $0.008 \mathrm{deg}$ for each kilogram of fuel asymmetry. Referring back to Fig. 2, the coning angle changed by approximately $0.016 \mathrm{deg}$ through seven maneuvers. This shift and the CM filter estimation results are discussed in the next section.

\section{Results}

The flight data used to test the CM filter consists of GPS Doppler shift measurements from 22 orbits for MMS2. This data is combined with attitude and rate estimates from the AGS Kalman filter used for generating the definitive attitude products and with MMS and GPS ephemerides.

It was found that the Doppler measurements are far noisier than those used for testing the CM filter before launch. Later investigation has shown that the Doppler errors are in agreement with prelaunch simulations, but these analyses were not known during development of the CM filter. The filter performance reported here is dominated by Doppler noise, and individual results are not robust to varying the filter noise parameter. There also is a significant uncertainty in the center-of-radiation for the individual GPS antennas, which introduces an unknown systematic error in Eq. 3.

To compensate for these errors, multiple data sets have been combined to increase the number of measurements, and a strong sigma-edit test is applied. That is, when each observed Doppler shift is compared with its predicted value from Eq. 13, this filter update is rejected if the difference (the observation residual) exceeds 1.5 times the $1 \sigma$ value expected from the estimated covariance matrix.

In addition, Doppler measurements are rejected if the GPS SV is more than 80,000 km from MMS. This has the effect of excluding weak signals and a large fraction of the signals coming from GPS side-lobes when MMS is near its apogee located well above the GPS constellation ( 20,000 km altitude). Doppler measurements are also rejected for four hours near perigee when the spacecraft velocity is largest and the attitude and spin rate are perturbed by the gravity-gradient torque.

Finally, the $1 \sigma$ fractional Doppler shift noise used in the CM filter has been taken to be $2 \times 10^{-8}$. This is a few orders of magnitude below the apparent actual noise. This level allows the filter to be responsive to the noisy signal without undergoing wild fluctuations. This type of ad hoc tuning would be unacceptable for an operational product, but for the current analysis, it is expected that the general trends can be determined by averaging over a large number of CM estimates and by considering only the shift in the CM offset as fuel is consumed rather than the CM itself.

It is expected that the CM filter requires between $10^{4}$ and $10^{5}$ updates for convergence, as seen in Fig. 1. Each MMS orbit is 24 hours long, and the Doppler data was available from up to 12 simultaneous SVs once every 30 seconds. For this reason, the 22 sample orbits have been grouped into seven multi-orbit data sets, each containing between 45,000 and 85,000 Doppler measurements.

These data sets are centered roughly on days 221 , $252,281,302,342$, and 362 of 2015 , and day 27 of 2016. These dates were chosen to fall between maneuvers. Formation maintenance maneuvers are performed in pairs on adjacent orbits. There is a single maneuver pair a few days before each of the seven data sets, with one exception where there are two maneuver pairs. Figure 3 shows an example of the filter results for the data set centered on day 281 .

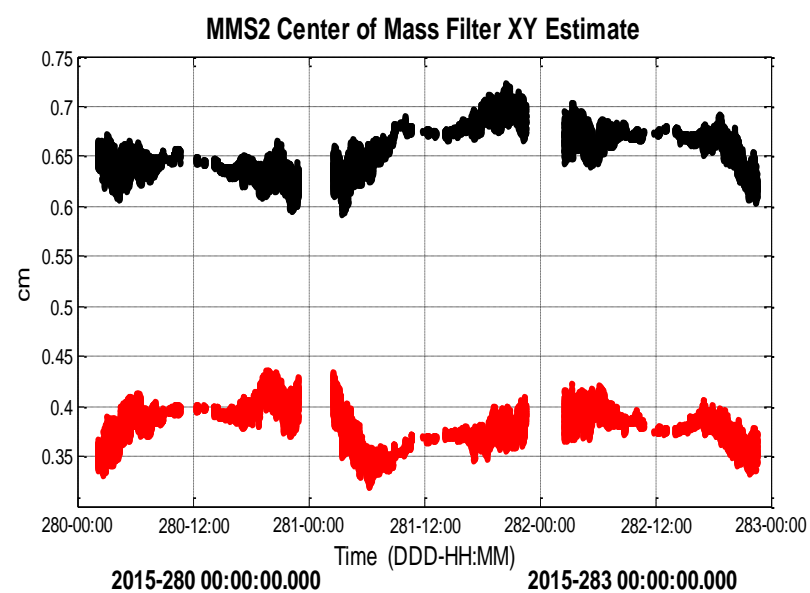

Figure 3. Filter estimates of the MMS body frame CM $X$ (red) and $Y$ (black) axes (in centimeters). 
The CM filter was run for each of the seven multi-orbit data sets. In each case, the mean CM was determined from the final 10,000 points. The overall change in the CM was determined by a linear fit over the seven data sets. The resulting total CM shift was found to be $-0.04 \mathrm{~cm}$ in the $X$-direction and $-0.16 \mathrm{~cm}$ in the $Y$-direction.

The CM shift implies a change in the mass distribution. As discussed in Sec. III, there are two known sources for mass asymmetry. First, even though the four tanks are designed to draw down equally, there may be some difference in the fuel consumed from each. Second, there may be a change in the shape of the fuel diaphragms. There are two known shapes the diaphragms can take during the fuel fill process. ${ }^{6}$ Two of the tanks are filled with propellant in the lower part of the tank, and two are filled with propellant in the upper part of the tank. When the fuel is in the lower part, the diaphragm takes on a roughly symmetric "mountain" shape. For the other two tanks, the fuel is in the upper part, and the diaphragm has an asymmetry, referred to as the "yin-yang" shape where the bulge of fuel behind the diaphragm is off-center. It is not clear how these shapes might relax or change as fuel is consumed. It is possible that this second effect was seen on MMS4 when, over a ten day period, the coning angle gradually changed by roughly 0.01 deg during a time when there were no maneuvers.

At the end of Sec. III, it was pointed out that the coning angle for MMS 2 changed by 0.016 deg through the seven maneuvers. The uncertainty in the coning angle estimate is determined from the covariance matrix of the attitude and rate filter. This $1 \sigma$ rate error is typically $0.002 \mathrm{deg} / \mathrm{sec}$ for the definitive attitude products generated daily by the AGS. With the spin rate of $18.6 \mathrm{deg} / \mathrm{sec}$, this implies a coning angle error of $0.006 \mathrm{deg}$.

If the 0.016 deg change in the coning angle is attributed to the fuel distribution, then Eqs. 16, 17, and 21 imply a change of the mass asymmetry of $2 \mathrm{~kg}$. For comparison, the fuel used during these seven maneuvers was 1.6, 8.8, 4.2, $2.5,0.8,2.2$, and $2.3 \mathrm{~kg}$. Section III also gave the result that the total spacecraft mass of $1354 \mathrm{~kg}$ and the roughly 1 meter tank-to-tank distance imply a $0.074 \mathrm{~cm}$ shift of the CM per kilogram of asymmetry. Thus, noting the sign of the coning angle shift, it is expected that the CM should shift by $-0.15 \mathrm{~cm}$, mainly in the $Y$-direction. This is in good agreement with the $-0.16 \mathrm{~cm}$ shift found from the CM filter.

\section{Conclusions}

This paper presented a review of two main results. The first was the design for the CM filter using the fractional Doppler shift of the GPS carrier frequency. The second was a relationship between mass asymmetry and the spacecraft coning angle. This review was followed by the application of these methods to MMS2 flight data.

Section IV discussed the uncertainty in the Doppler data and the measures taken in the filter to control these errors. The methods included the use of large merged data sets, a tight sigma-edit tolerance, discarding data near perigee and from SVs that may have weak signals, and unphysically adjusting the filter noise parameter. Finally, the overall CM trend was obtained by fitting the results from the seven data sets.

The coning angle analysis showed that an apparent mass asymmetry of $2 \mathrm{~kg}$, separated by roughly one meter, had developed over the course of seven maneuvers. If the same mass asymmetry affects the CM, then a shift of $-0.15 \mathrm{~cm}$ is expected. Application of the CM filter yielded a shift of $-0.16 \mathrm{~cm}$. The consistency between the coning angle and CM filter estimate validates the proposed GPS Doppler shift method for estimation of the CM on spinning spacecraft.

\section{Acknowledgments}

The author would like to acknowledge the suggestions and contributions from other members of the MMS Flight Dynamics team, especially Anne Long and Emil Superfin. This work was supported by the National Aeronautics and Space Administration (NASA)/Goddard Space Flight Center (GSFC), Greenbelt, MD, USA, Contract NNG14VC09C.

\section{References}

${ }^{1}$ Farahmand, M., Long, A., and Carpenter, R., "Magnetospheric Multiscale Mission Navigation Performance Using the Goddard Enhanced Onboard Navigation System," 25 th International Symposium on Space Flight Dynamics, Munich, Germany, Oct., 2015.

${ }^{2}$ Sedlak, J. E., Superfin, E. A., and Raymond, J. C., "Magnetospheric Multiscale (MMS) Mission Attitude Ground System Design," $22^{\text {nd }}$ International Symposium on Spaceflight Dynamics, INPE, São José dos Campos, SP, Brazil, Feb., 2011.

${ }^{3}$ Queen, S. Z., "A Kalman Filter for Mass Property and Thrust Identification of the Spin-Stabilized Magnetospheric Multiscale Formation," $25^{\text {th }}$ International Symposium on Spaceflight Dynamics, Munich, Germany, Oct., 2015.

${ }^{4}$ Raymond, J. C., Sedlak, J. E., and Vint, B., "Attitude Ground System (AGS) for the Magnetospheric Multiscale (MMS) Mission," $25^{\text {th }}$ International Symposium on Spaceflight Dynamics, Munich, Germany, Oct., 2015.

${ }^{5}$ Markley, F. L. and Sedlak, J. E., "Kalman Filter for Spinning Spacecraft Attitude Estimation," Journal of Guidance, Control, and Dynamics, Vol. 31, No. 6, p. 1750, Nov.-Dec. 2008.

${ }^{6}$ Mariconti, S., "Magnetospheric Multiscale (MMS) Project Mass Properties Report,” NASA/GSFC, 461-MECH-RPT-0086, 2013. 